Perspective

\title{
The Machine-to-Everything (M2X) Economy: Business Enactments, Collaborations, and e-Governance
}

\author{
Benjamin Leiding ${ }^{1, *,+}\left(\mathbb{D}\right.$, Priyanka Sharma ${ }^{1,+}+_{(\mathbb{D}}$ and Alexander Norta ${ }^{2,+}(\mathbb{D}$ \\ 1 Institute for Software and Systems Engineering, Clausthal University of Technology, \\ 38678 Clausthal-Zellerfeld, Germany; priyanka.sharma@tu-clausthal.de \\ 2 Department of Software Science, Tallinn University of Technology, 12616 Tallinn, Estonia; \\ alexander.norta@taltech.ee \\ * Correspondence: benjamin.leiding@tu-clausthal.de \\ + These authors contributed equally to this work.
}

Citation: Leiding, B.; Sharma, P.; Norta, A. The Machine-to-Everything (M2X) Economy: Business Enactments, Collaborations, and e-Governance. Future Internet 2021, 13, 319. https://doi.org/10.3390/ fi13120319

Academic Editor: Paolo Bellavista

Received: 7 December 2021

Accepted: 13 December 2021

Published: 19 December 2021

Publisher's Note: MDPI stays neutral with regard to jurisdictional claims in published maps and institutional affiliations.

Copyright: (C) 2021 by the authors. Licensee MDPI, Basel, Switzerland. This article is an open access article distributed under the terms and conditions of the Creative Commons Attribution (CC BY) license (https:// creativecommons.org/licenses/by/ $4.0 /)$.
Abstract: Nowadays, business enactments almost exclusively focus on human-to-human business transactions. However, the ubiquitousness of smart devices enables business enactments among autonomously acting machines, thereby providing the foundation for the machine-driven Machineto-Everything (M2X) Economy. Human-to-human business is governed by enforceable contracts either in the form of oral, or written agreements. Still, a machine-driven ecosystem requires a digital equivalent that is accessible to all stakeholders. Additionally, an electronic contract platform enables fact-tracking, non-repudiation, auditability and tamper-resistant storage of information in a distributed multi-stakeholder setting. A suitable approach for M2X enactments are electronic smart contracts that allow to govern business transactions using a computerized transaction protocol such as a blockchain. In this position paper, we argue in favor of an open, decentralized and distributed smart contract-based M2X Economy that supports the corresponding multi-stakeholder ecosystem and facilitates M2X value exchange, collaborations, and business enactments. Finally, it allows for a distributed e-governance model that fosters open platforms and interoperability. Thus, serving as a foundation for the ubiquitous M2X Economy and its ecosystem.

Keywords: blockchain; smart contract; M2X; smart autonomous devices; e-governance; lifecycle management

\section{Introduction}

An open Machine-to-Everything (M2X) Economy [1] emerges when humans and smart autonomous devices interact, transact, and collaborate, e.g., self-driving buses and autonomous food delivery in a smart-city context $[2,3]$. The ubiquitousness of smart devices also allows for business transactions without human intervention among autonomously acting machines. Besides Machine-to-Machine (M2M) interactions, machines interact with humans (Machine-to-Human-M2H), or infrastructure components (Machineto-Infrastructure-M2I) - combined they provide the foundation for the machine-driven M2X Economy. While related concepts such as the Internet of Things (IoT), Smart Homes as well as Smart Cities [4], and the Industry 4.0 [5] have evolved, they do not support an interoperable, integrated, scalable model that facilitates the M2X Economy. Likewise, concepts for M2X value transfer, collaborations, and distributed e-governance are missing to achieve the shared objectives. Moreover, integrating humans and smart devices into a well-functioning socio-technical system [6] is essential, as it puts the M2X concept in a human-centered context.

In the M2X Economy, smart sensors may offer collected sensor data such as temperature, or air contamination to interested buyers that rely on the aforementioned data for their own computations. In the context of autonomous and self-driving vehicles, scenarios such as automated tollbooth payments, autonomous battery charging services as well as general 
Transportation-as-a-Service (TaaS) applications are among the most discussed use cases [7]. Thus, a socio-technical business model is required as it facilitates the M2X Economy.

Various M2X-resembling applications and use cases already exist, e.g., in the context of IoT. However, complex and impactful applications are still missing that provide more than marginal value to society. In addition, an economy emerging from M2X enactments among humans, smart devices, software agents, and physical systems is rarely considered. To provide or utilize non-trivial services, smart devices may also have to collaborate ondemand with other entities to be able to achieve a shared goal, or even migrate to different geographical locations based on supply and demand. Accordingly, "the interleaved ondemand collaborations, interactions, and transactions among autonomous, heterogeneous, and highly dynamic entities (humans, machines, software agents, etc.) lead to a decentralized, distributed and heterogeneous socio-technical system consisting of a large number of micro-services of different vendors and solution as well as infrastructure providers" [1].

This trend coincides with the emergence of smart-contract blockchain technology [8] that allows for novel peer-to-peer (P2P) electronic governance models. Traditionally, human-to-human business enactments are governed by contracts either in the form of oral, or written agreement. A machine-driven ecosystem requires a digital equivalent that is accessible to all stakeholders, i.e., a smart contract-driven platform that allows for fact tracking, non-repudiation, auditability, and tamper-resistant storage of information in a distributed multi-stakeholder setting. Electronic smart contracts enable and govern business transactions using a computerized transaction protocol such as a blockchain. Moreover, smart-contract blockchain technology comprises computer programs for the consistent execution by a network of mutually distrusting nodes where no arbitration of a trusted authority exists.

A one-stop platform for the provision and enactment of services and goods of a M2X ecosystem is desirable instead of a manufacturer-focused platform with deliberately forced, or functional lock-ins that lead to the formation of self-contained data and service silos such as Tesla, Google, or Amazon. Instead, an interoperabilty layer that implements the compatibility of different manufacturer platforms is required to allow for the exploitation of economies of scale and increased efficiency. Thus providing the foundation for an ecosystem that can be operated as a joint venture of various stakeholders and includes builtin e-governance mechanisms, thereby constituting a neutral territory for all stakeholders.

In this position paper, we argue in favor of an open, decentralized and distributed smart-contract-based M2X Economy that supports the corresponding multi-stakeholder ecosystem and facilitates M2X value exchange, collaborations, and business enactments. Furthermore, the M2X Economy allows for a distributed e-governance model that fosters open platforms and interoperabilty. To do so, we draw from a variety of previous work and assemble an initial set of essential building blocks for a future M2X Economy and its corresponding ecosystem.

The research methodology of this work follows the usual approach of a position paper: First, we stipulate our position by presenting an innovative hypotheses-as stated above, we argue in favor of an open, decentralized, and distributed smart-contract-based M2X Economy. Subsequently, related background information pertaining to the position are provided. Second, we provide evidence to support our position. Third, we follow a discussion of both sides of the matter before concluding the presented position statement.

Our position paper provides three main contributions: First, it is a call for a discussion of an emerging machine-driven economy and its corresponding ecosystem with autonomously acting devices offering and consuming services in a M2X context. Second, it suggests a course of actions for developing the M2X Economy needs to focus on specific domains. Third, it outlines enabling concepts of the M2X Economy.

The remainder of this paper is structured as follows: Section 2 introduces the M2X Economy in detail, showcases the state of the art, and discusses related work. Next, Section 3 focuses on mechanisms for M2X stakeholders to interact, transact, and collaborate by means of a smart-contract-based lifecycle approach and a corresponding distributed 
e-governance infrastructure. Section 4 details the smart token economics. Subsequently, Section 5 discusses our position as well as alternative approaches. Finally, Section 6 concludes our work.

\section{The M2X Economy}

The evolving M2X applications and the corresponding ecosystem will influence our daily lives in many ways. Besides M2M interactions, machines interact with humans (M2H) or infrastructure components (M2I). The framework of the M2X Economy represents a more general view on use cases that involve autonomous smart devices and also encompasses $\mathrm{M} 2 \mathrm{M}, \mathrm{M} 2 \mathrm{H}$, and M2I scenarios [1].

In Section 2.1 we first present the running case that is used for illustration purposes throughout this work. Afterwards, Section 2.2 introduces related concepts such as cybernetics, IoT, cyber-physical systems (CPS), and wireless sensor networks (WSNs) as well as related work. Next, is the definition and elements of the M2X Economy in Section 2.3.

\subsection{Running Case}

We introduce an example running case of the M2X Economy in order to provide the reader with a better understanding as well as the scope of M2X applications. The selected running case is illustrated in Figure 1 and belongs to the sub-set of vehicle-focused M2X applications, i.e., the vehicle-to-everything (V2X).

In the future, people might not possess vehicles any more. Instead, vehicles may own themselves, or they are owned by the government, or private corporations [1]. We assume that Alice requests a self-driving car (TaaS) to go from Point $A$ to $B$ and several route options exist for this. Figure 1 indicates that the fastest route option is expensive but also the most comfortable and equipped with toll gates. Alternatively, the less comfortable, cheaper option is via Point $C$ and includes traffic lights and traffic congestion. Alice may select her preferred option depending on her price range and on the urgency of reaching Point $B$. Furthermore, we assume that the self-driving cars are able to communicate with each other as well as the traffic lights (infrastructure). It is also possible to buy a green-light phase for a faster commute to Point $B$. Finally, Figure 1 shows an electric charging station near Point $B$ that the self-driving cars may use for some amount of fee. In the described running case, assuming that time and money are important factors, Alice may select from a range of possible options. On the one hand, she may choose the fastest and most expensive route to Point $B$, or take the less comfortable and cheaper option via Point $C$. Additionally, she can pay an extra fee and her car may negotiate for a green light at the traffic signals.

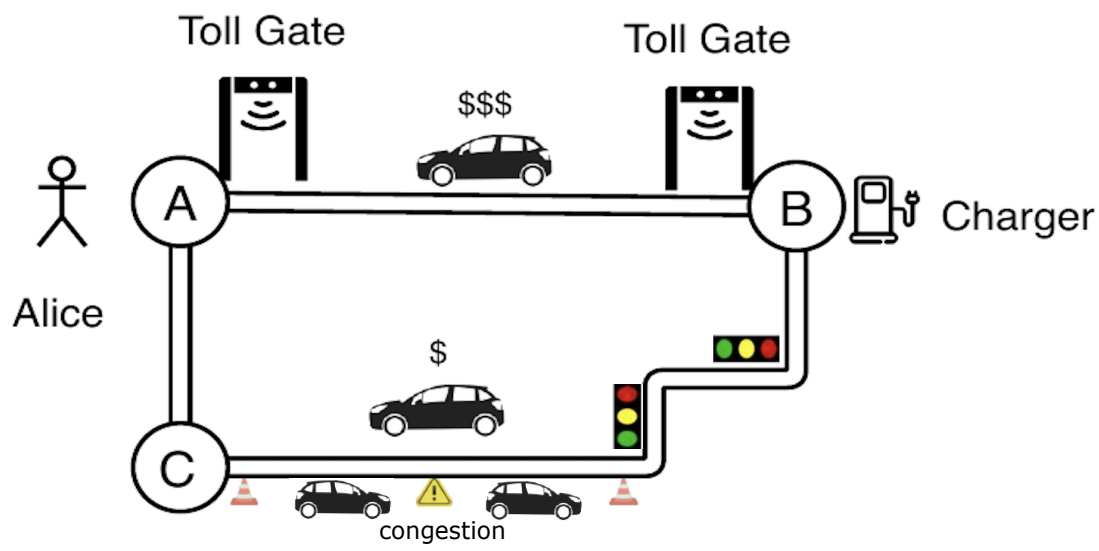

Figure 1. Self-driving M2X running case incorporating smart traffic lights and a traffic-congestion response, adapted from [1].

Our running case-despite it simplicity—already covers a wide variety of M2X service enactments, i.e., TaaS, toll gate payments, battery electric vehicle (BEV) charging, road 
space negotiations, smart parking, and traffic information provision. Nevertheless, they also only constitute a small subset of services within the M2X ecosystem.

\subsection{State of the Art and Related Work}

The idea of the M2X Economy and its ecosystem overlaps with some closely related concepts and applications such as cybernetics, WSNs, CPS, and IoT [1]. This section clarifies the differences and overlaps with those concepts and applications.

Wiener [9] defines the concept of cybernetics as "the scientific study of control and communication in the animal and the machine", while WSNs consist of spatially distributed autonomous sensors to monitor physical or environmental conditions and to cooperatively pass their data through a variety of networks to a main location [10].

CPS are engineered systems that are built from, and depend upon, the seamless integration of computation and physical components. CPS tightly integrate computing devices, actuation, and control, networking infrastructure, and sensing of the physical world [11].

Gubbi et al. [12] defines IoT as an "interconnection of sensing and actuating devices providing the ability to share information across platforms through a unified framework, developing a common operating picture for enabling innovative applications. This is achieved by seamless, large-scale sensing, data analytic and information representation using cutting edge ubiquitous sensing and cloud computing".

Robotic Process Automation (RPA) is regarded as one of the most advanced technologies in the area of computers science, electronic and communications, mechanical engineering, and information technology [13]. With software robots autonomously executing their choreography uninterruptedly, quickly, and flawlessly while at the same time being easy to implement at relatively low costs compared to traditional process automation, RPA may automate processes enabling business transactions in the near future [14].

After clarifying the terms and concepts above, the question remains: Where does the M2X Economy fit in? Several publications list and survey CPS and IoT applications, e.g., [15-19]), as well as their economic value and impact, e.g., [19-21]. However, the emerging economy resulting from M2X enactments among humans, smart devices, software agents and physical systems is rarely considered.

\subsection{Elements and Definition of the M2X Economy}

The M2X Economy framework involves autonomous smart devices and further encompasses mobile devices, software agents, humans, and infrastructure in $\mathrm{M} 2 \mathrm{M}, \mathrm{M} 2 \mathrm{H}$, and M2I scenarios. A main requirement of such an ecosystem is to enable a seamless integration of humans and smart devices into a well functioning socio-technical system that puts the M2X concept in a human-centered context [1]. When considering collaborations and interactions between the M2X stakeholders, multilevel and unidirectional interrelations can be seen. The interleaved on-demand collaborations, interactions and transactions among autonomous, heterogeneous and highly dynamic entities (humans, machines, software agents, etc.) lead to decentralized and distributed socio-technical systems comprising a large number of micro-services of different vendors and solutions, as well as infrastructure providers [1].

Definition 1. Thus, the M2X Economy is the result of interactions, transactions, collaborations and business enactments among humans, autonomous and cooperative smart devices, software agents, and physical systems. The corresponding ecosystem is formed by automated, globallyavailable, heterogeneous socio-technical e-governance systems with loosely coupled, P2P-resembling network structures and is characterized by its dynamic, continuously changing, interoperable, open and distributed nature. Thereby, the M2X Economy employs concepts such as cyber-physical systems, the Internet of Things, and wireless sensor networks. 


\section{Enactment, Collaboration, and e-Governance}

Human-to-human business enactments are governed by enforceable contracts either in the form of an oral, or written agreement. Contract documents [22] uniquely identify the contracting parties, the offered services, or goods, a corresponding compensation, as well as further constraints such as delivery dates, quality goals, penalties, and means of arbitration [23]. Still, a highly-automated and machine-driven ecosystem requires a digital equivalent that is accessible to and usable by all stakeholders. Moreover, traditional solely human-focused contracts are often under-specified and thus, not suitable for M2X enactments [23]. "Most importantly, traditional contracts do not provide sufficient details about the actual transaction process, and consequently, frictions between the contracting parties are very likely, e.g., one party assumes a specific product certificate before delivering a partial compensation, and the other party assumes the opposite" [23].

Electronic smart contracts $[24,25]$ address the listed issues by enabling and governing business transactions using a computerized transaction protocol such as a blockchain. Blockchain technology [26] ensures a trustworthy, tamper-resistant, P2P transaction processing, and enables a distributed, often decentralized, transparent way for communication. More generally, a blockchain is a distributed ledger that enables users to send data, process it, and verify it without the need for a central entity [26]. In addition, smart-contract blockchain technology comprises computer programs for the consistent execution by a network of mutually distrusting nodes where no arbitration of a trusted authority exists. As a result, allowing for fact tracking, non-repudiation, auditability, and tamper-resistant storage of information in a distributed multi-stakeholder setting.

On the one hand, the running case of Section 2.1 only presents a small fraction of potential applications and use cases of the M2X Economy. On the other hand, the running case already contains several examples of different M2X interactions, transactions, and collaborations, i.e., TaaS, road space negotiations, toll gate payments, BEV charging, traffic light information dissemination, and smart parking. The enactments of the listed examples follow a similar process structure, thus allowing for an abstraction towards a general lifecycle of the M2X Economy. Consequently, we stipulate that all M2X-related interactions, transactions, collaborations, and further enactments can be governed and represented using a blockchain-based smart contract.

In the following, Section 3.1 details a conceptual lifecycle for M2X business enactments and collaborations using electronic smart contracts. Afterward, Section 3.2 outlines corresponding distributed e-governance mechanisms.

\subsection{Digital Contract Lifecycle Management}

Based on [23], Norta presents a conceptual smart contract-based lifecycle as illustrated in Figure 2.

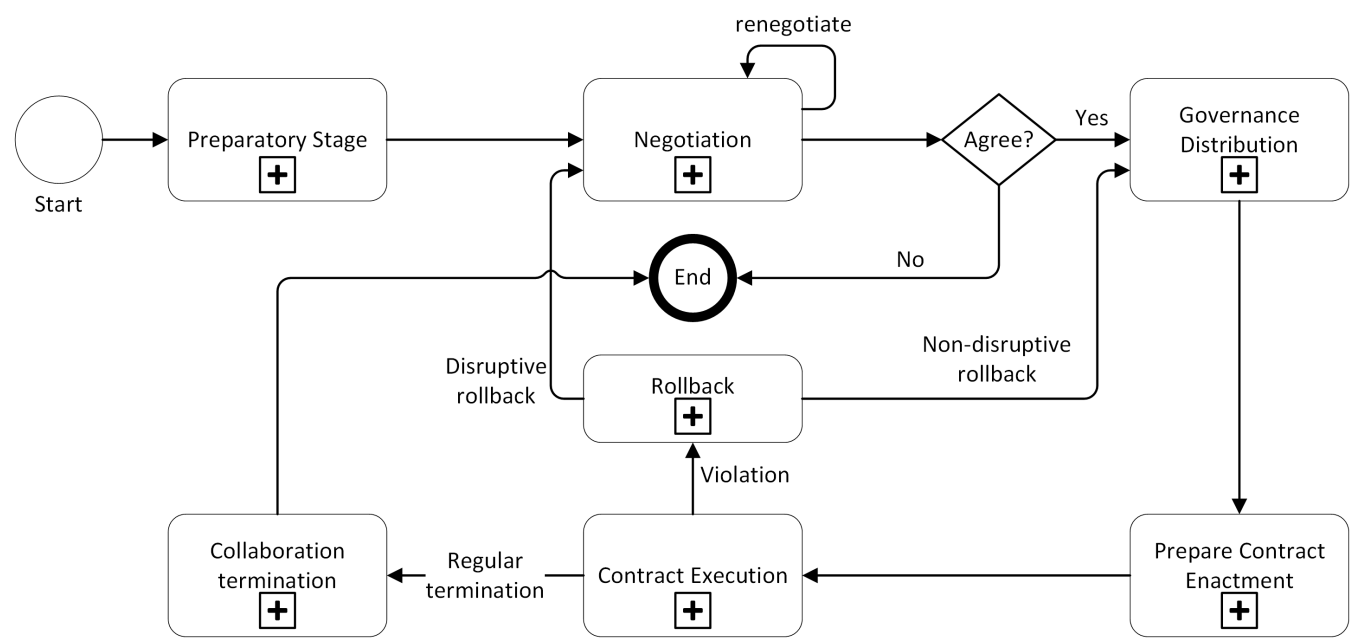

Figure 2. Conceptual lifecycle for M2X business enactments-Based on [1,23]. 
The lifecycle is divided into seven stages: (i.) preparation, (ii.) negotiation, (iii.) governance distribution $(i v$.) preparation of collaboration enactment $(v$.) collaboration enactment (vi.) rollback, and (vii.) termination stage.

The preparatory stage is initiated by selecting a pre-configured template from a distributed service hub. The distributed service hub hosts contract templates that match different M2X use-cases and outlines the corresponding contractual process flow. Following the running case, a template for TaaS is selected and populated with information about the involved entities, such as identifiers and wallet addresses. Moreover, TaaS-specific conditions are defined, e.g., departure location, final destination, the required vehicle size, and the departure/arrival time. Subsequently, the TaaS contract request is negotiated with potential TaaS service providers, i.e., autonomous vehicles. The negotiated-contract conditions primarily depend on information such as the travel distance and energy consumption of the vehicle as well as the number of transported individuals.

The negotiation stage concludes either with an agreement-resulting in a contract signed by both parties to express their approval—or a contract rollback if no agreement is reached. In our case, Alice and the vehicle serving the direct route between A and B agree upon a set of rights and obligations. Subsequently, a smart contract is established and serves as a distributed governance infrastructure (DGI) coordinating agent (also see Figure 3). Finally, the e-governance distribution commences, Alice and the vehicle each receive local contract copies containing the respective obligations and rights of each party resulting from the previous negotiations [23]. The vehicle's and Alice's obligations are observed by monitors and are assigned so-called business-network model agents (BNMA) that connect to IoT-sensors such as the vehicle's GPS-sensor [23].

The required process endpoints, e.g., for payment processing as Alice pays using the cryptocurrency of her choice, are prepared and provided as part of the contract enactment preparation. "Once the e-governance infrastructure is set up, technically realizing the behavior in the local copies of the contracts requires concrete local electronic services. After picking these services, follows the creation of communication endpoints so that the services of the partners are able to communicate with each other. The final step of the preparation is a liveness check of the channel-connected services" [23].

Next, the contract execution stage is triggered, and the vehicle picks up Alice at location A. The TaaS contract enactment terminates, or expires once Alice arrives at Point B. Alternatively, the contract is prematurely terminated, e.g., failing to transport Alice to Point $\mathrm{B}$, or violating agreed upon time restrictions, might result in an immediate rollback of the TaaS contract, or invokes a mediation process that is supervised by a conflict-resolution escrow service that is not depicted in Figure 2. Note that the enactment of the TaaS running case subsumes further M2X enactments that occur throughout the TaaS service provision, e.g., the vehicle pays a minor fee at the toll gate to use the faster toll road. The toll road payment is part of the costs to transport Alice from Point A to B and is thus, included in her fare.

\subsection{Distributed e-Governance}

While Figure 2 presents the collaboration among partners from a lifecycle perspective, Figure 3 depicts the creation sequence of a DGI from an infrastructure perspective, thereby providing the foundation for a distributed, interoperable, dynamic ad-hoc enactment among heterogeneous M2X entities. 
a) Distributed Governance Infrastructure

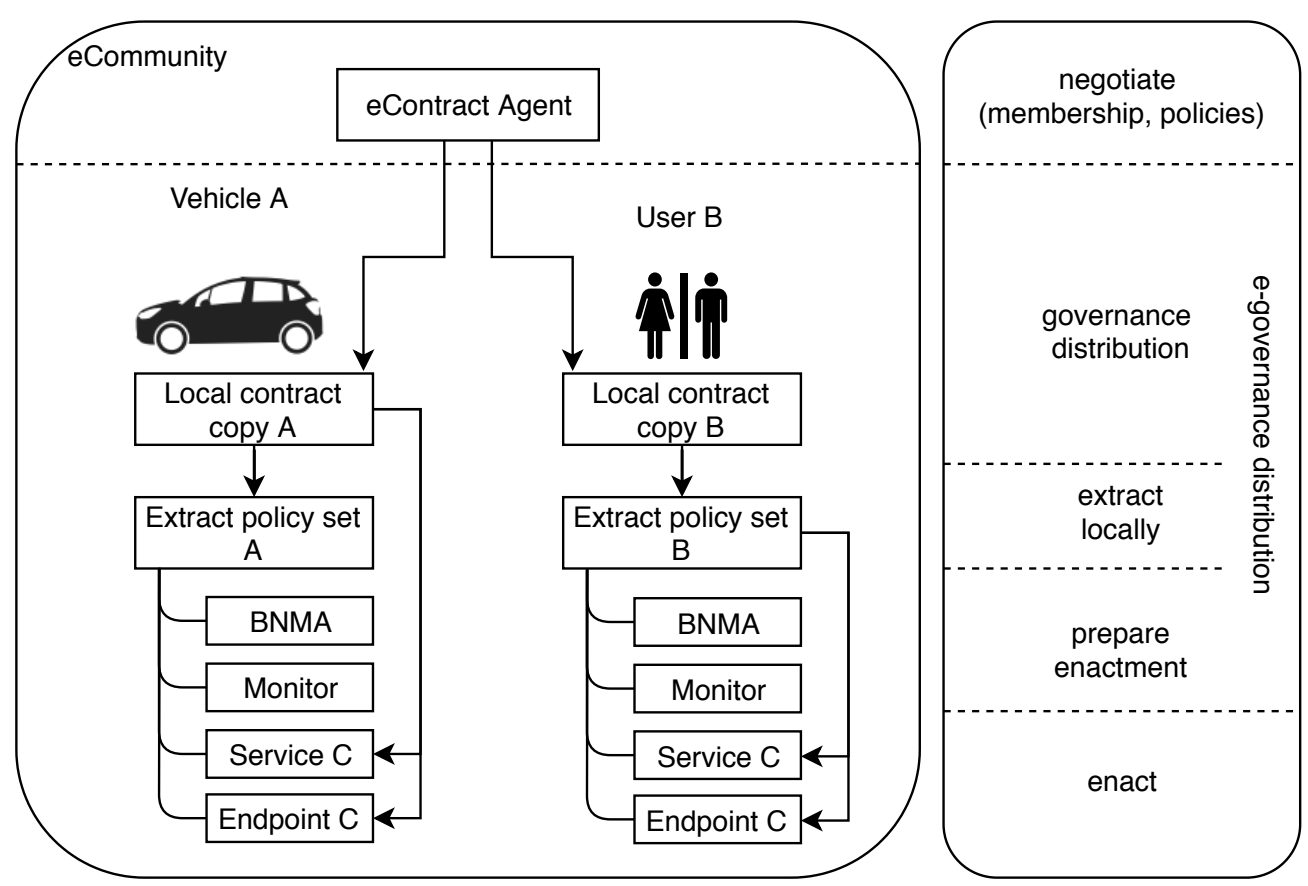

Figure 3. Distributed M2X governance infrastructure. Source: [1] and based on [23,27].

Finally, the M2X collaboration model enables providers to decide if and in which way changes to a private and internal process must be projected to a related public process view in a way where the process view and the internal process stay consistent with each other. Thus, the M2X collaboration model enables service-consumers to monitor a public process view to safely follow changes performed to a private and internal process.

This way, it is possible to support the evolution of smart contracts [28] as a significant means to achieve flexibility in B2B collaborations. As smart contracts are instrumental to enable decentralized autonomous organizations (DAO) [23] for the formation of electronic communities, service-oriented cloud computing (SOCC) [29] supports companies in the coordination of information- and business-process flows [30] for the choreography and orchestration [31] of heterogeneous legacy-system infrastructures.

For evolving DAO-collaborations, Figure 4a shows a conceptually collaboration configuration where the template for an electronic-community formation is given by a businessnetwork model (BNM) [32] to specify choreographies relevant for a respective business scenario.The BNM defines legally valid [33-35] template contracts as service types together with assigned organizational roles. A collaboration hub that houses business processes as a service (BPaaS-HUB) [36] in the form of process views [30], houses the BNM templates for potential collaborating counterparties to enable a speedy matching.

The external layer of Figure 4 a depicts service offers to identically match the service types defined in the BNM with the respective collaborating partner contractual sphere. Furthermore, a collaborating partner is required to comply with a specific partner roles assigned to a specific service type. In [30], further details are contained about a tree-based process-view matching for creating DAO-configurations. We stress that Figure 4a uses Petri net [37] notation, which can be mapped into a tree-formalization as well with less computationally expensive strain.

Figure $4 \mathrm{~b}$ presents a corresponding mapping and presents the top-level structure of a smart contract using the eSourcing Markup Language (eSML) [38]. "The core structure of a smart contract we organize according to the interrogatives Who for defining the contracting parties together with their resources and data definitions, Where to specify the business and legal context, and What for specifying the exchanged business values. For achieving 
a consensus, we assume the What-interrogative employs matching process views that require cross-organizational alignment for monitorability" [23].

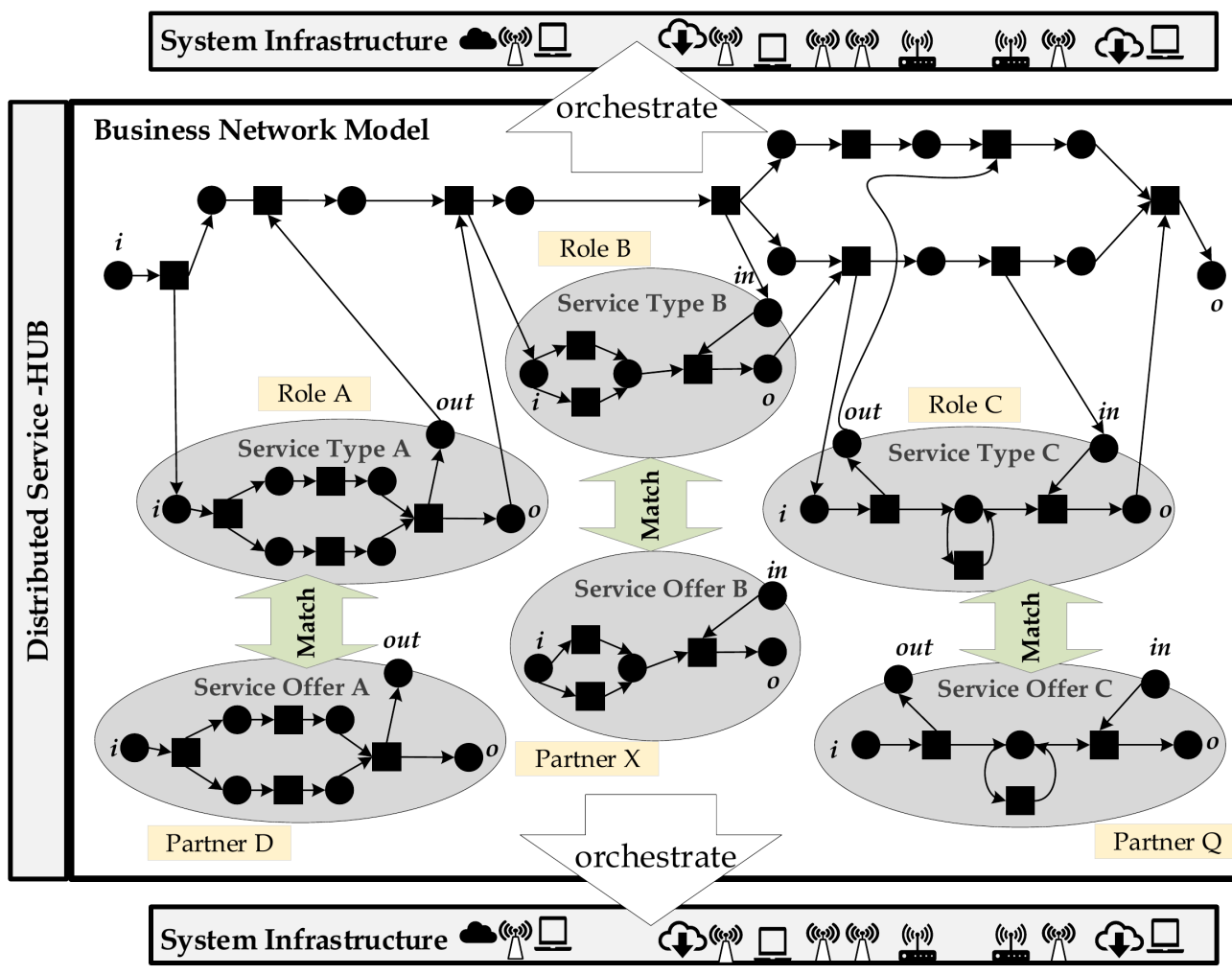

(a)

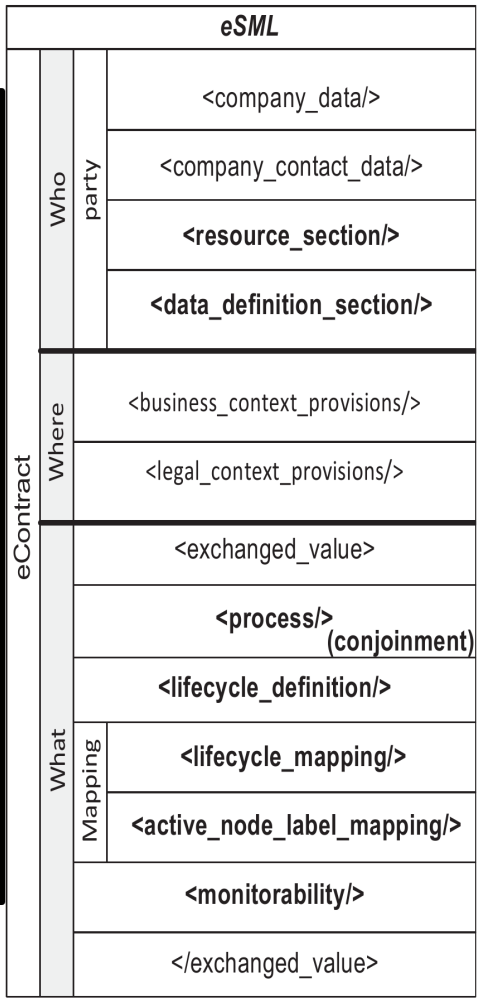

(b)

Figure 4. (a) P2P service matching and provision of the M2X ecosystem using the eSourcing framework-(Based on) [23]. (b) The eSourcing Markup Language (eSML) for specifying contractual collaborations-Based on [38].

\section{Smart Token Economics}

The running case of Section 2.1 shows that the M2X Economy is a complex, distributed, and socio-technical framework that requires a novel approach for developing the monetary economy. We infer that the traditional financial system is not suitable and lacks the utility for consideration in the M2X Economy. An important reason is that an integration of the financial legacy technology does not scale and perform for a context such as the running case in Figure 1 and additionally, to technically support the incentives mechanisms between the human user termed Alice and the smart autonomous devices being the cars, traffic lights, toll gates, and charging stations, we require programmable monetary units, which fiat-currencies are not, e.g, as a code extension of an ERC20-token smart-contract template (https: / / eips.ethereum.org/EIPS/eip-20 accessed on 4 November 2021). Consequently, the novel domain of token economics [39] emerges to compensate for the deficiencies of the legacy fiat-currency system. Informally, a token economy in an M2X Economy that employs smart-contract blockchain technology, is characterised by encouraging desirable behavior by the human and artificial agents and infrastructure involved by offering rewards and optionally also penalties in the form of crypto tokens.

We stress that established schools of thought of economics do not typically assume that a monetary unit is programmable and connected as such to a socio-technical application system context as Section 2.1 describes, where the automated complex governance of incentives mechanisms is essential for P2P interactions between humans, smart autonomous devices, and infrastructure. On the other hand, a set of standard-token smart contracts are available, initially offered by Ethereum, that allow for flexible instantiations into diverse token types [40], e.g., tokens for a platform, that play a role of a security, or facilitate transactions, enable specific platform-utility use, e-governance tokens for complex voting 
mechanisms, reputation tokens, and so on (https:/ / tinyurl.com/token-types accessed on 10 November 2021).

As token economics based on smart-contract blockchain technology is an emerging computer-science driven scientific discipline, we infer that the programmable nature of crypto tokens requires a novel development methodology that is integrated with the M2X system design from the very inception. In earlier research [41], we discover that no suitable methodology exists for developing blockchain distributed applications (DApps), which is relevant too for an M2X context. Consequently, the distributed agent-oriented modeling (DAOM) method [42] fills this gap, being the first blockchain-DApp development method that also integrates the foundation for the development of a DApp-specific token economy being integrated with the system functionalities.

While due to page limitations, we refer interested readers to several use cases [43,44], where the DAOM method follows a set of briefly described model-driven design steps. First, the functional and quality goals, together with human and artificial software agents are organized into a so-called goal model where transparent gray rectangles with tokentype labels denote smart-contract blockchain application in a DApp. Next, based on a set of heuristics, a component-diagram architecture is deduced from the goal model where blockchain-involving components are also gray colored, corresponding to the specific requirements of derivation. The addition in the component-diagram architecture is the specification of the information-exchange channels between components, and components to human and artificial software agents. Based on this conceptual DApp understanding, DAOM next prescribes the specification of so-called on-chain transaction sets that are a tuple comprising an ID, short description and agents involved per respective transaction evaluation. It is important to specify this on-chain transaction set given the expenses of transaction validations [45], e.g., per proof-of-work (PoW), proof-of-staking (PoS), and so on. Finally, the set of information-exchange protocols between components, and components with human and artificial software agents, is expressed either in sequence diagrams, or in a graph-based notation such as business process model and notation (BPMN) [46] in which the IDs of respective on-chain transactions are embedded.

Note that the DAOM method is inherently technology agnostic and allows subsequently for deducing a technology stack with a considerable blockchain subset for a detailed token-economics establishment to govern the incentive mechanisms and a rapid Dapp development. At the same time, extension work is required to develop DAOM further for full applicability in an M2X context. More concretely, since smart autonomous devices are an essential part of M2X, being software agents embedded in hardware, further modeling notations must be adopted into the DAOM method for designing specifically the behavior of the $\mathrm{P} 2 \mathrm{P}$-communicating smart autonomous devices and also the smart-contract instantiations that constitute the respective token types to govern the incentive mechanisms. A promising option is to consider agent-based computational economics [47] in combination with a future extended DAOM method for M2X-focused smart-token economics development.

\section{Discussion}

The previous Section 2 introduces the M2X Economy, while Sections 3 and 4 focus on essential building blocks of the M2X Economy, i.e., M2X enactments, governance and smart-token economics. Subsequent sections discuss the arguments in favor and against our smart-contract enabled and blockchain-based M2X proposal as well as alternative approaches. Space constraints force us to focus on the most relevant aspects.

\subsection{Digital Smart Contracts}

While human-to-human business enactments are governed by oral, or written contracts, they are not applicable to the highly-automated, machine-driven and human-focused M2X Economy. First, human-centered oral and written contracts are difficult to process even for smart machines [1]. Second, traditional contracts [48] are often under-specified and do not provide sufficient details about the actual transaction processes as well as 
about the parties obligations and rights $[23,34]$. Third, they do not allow for extensive automation, scale badly and lack a computerized transaction protocol [49]. Fourth, efficient and automated means of conflict-resolution are missing [1,23].

While we propose the utilization of electronic smart contracts to address the issues above, one may argue that a cloud-based online shop for services of the M2X Economy would be sufficient, e.g., Amazon's web shop proves to scale well and even partially automates business enactments. Still, such types of business enactments suffer from transparency issues which complicate—or even prevent and sabotage-conflict-resolution mechanisms. Especially the unequal power relations between a single entity and the service-offering cloud shop prevent fair markets and business enactments.

In contrast, smart contracts allow for the automated, consistent, transparent, and auditable enactment of contracts by a network of mutually distrusting nodes where no arbitration of a trusted authority is required [24,50,51]. As a result, allowing for fact tracking, non-repudiation, auditability, and tamper-resistant storage of information in a distributed multi-stakeholder setting. In case of any conflicts, pre-defined rollback mechanisms are applied as described in [23].

Finally, Amazon-resembling service provision promotes lock-in effects, and obstructs much needed interoperability and openness of the M2X ecosystem as discussed in the subsequent Section 5.2. Neither traditional contracts, nor a cloud-hosted shop-resembling service provisions, allow for dynamic, P2P- (even local) ad-hoc enactments.

\subsection{Openness and Interoperability}

A one-stop platform for the provision and enactment of services and goods of a M2X ecosystem is desirable instead of a manufacturer-focused platform with deliberately forced, or functional lock-ins that lead to the formation of self-contained data and service silos such as Tesla, Google, or Amazon. As suggested in [1], interoperability allows for the exploitation of economies of scale and increased efficiency. At the same time, an interoperable blockchain ecosystem can be operated as a joint venture of various stakeholders and include built-in e-governance mechanisms, thereby constituting a neutral territory for all stakeholders $[1,52]$. A smart-contract driven M2X platform and its corresponding ecosystem not only enable an interoperable platform for M2X entities, but also further reduces dependency on intermediaries [53].

The technical implementation is realized by so-called relay chains as introduced by Polkadot [52] that provide communication interfaces for different heterogeneous blockchain platforms to interact with each other and subsequently, allow for a blockchain-agnostic, highly-automated, globally-available orchestration and choreography of heterogeneous socio-technical systems. Thus, specific manufacturers, or service-provider specific functionalities may also be accessible outside their own platform.

\subsection{Identity}

In order for hardware devices, humans and software agents to conduct digital business transactions, or enact digital collaborations as described in Section 2.1, all these entities require a digital representation of their "real-world" identity. To enable secure business collaborations and transaction within the M2X Economy, this digital representation is required to establish and enable trust, reputation mechanisms, perform verifiable and accountable transactions, and establish reliable as well as auditable data provenance [1]. As M2X is a multi-stakeholder ecosystem, the identity management issue applies not only for its users, but also infrastructure providers, OEMs, regulators and service providers. A single central authority for identity management of all these different stakeholders poses the risk of single point of failure. Furthermore, identity silos create privacy concerns and are not interoperable [54].

As earlier argued in this section, centralized infrastructures are not suitable for facilitating the full potential of the M2X ecosystem. Hence, a centralized identity solution is not an option and a decentralized interoperable identity solution is required. In order 
to prevent the aforementioned flaws and enable an open interoperable ecosystem, the identity-management solution needs to be self-sovereign and user-centric. Self-sovereign identity puts end-users in charge of decisions about their own privacy and disclosure of their personal information and credentials [54] and not the organizations that traditionally centralize identity. Self-sovereign identity systems that are based on decentralized identifiers (DIDs) [55], utilize distributed ledgers, or blockchains as a distributed storage system that replace centralized and incompatible data silos with a cooperative shared storage resource. The result is a user-controlled identity provision model where users control access and sharing of their data based on a need-to-know-basis using the concepts of DIDs, DID documents, and verifiable claims [1].

\subsection{Trust}

Blockchains are trust engines in an inherently trustless M2X Economy collaboration context. Blockchain technology promises to secure the M2X ecosystem where the management of large and distributed datasets in a secure way is essential. Still, the expected performance and scalability of existing blockchains is currently not compatible for a M2X context [56]. Consequently, new types of blockchains with novel consensus and validation algorithms are required for the large number of securely connected smart autonomous devices that interact with other machines, humans, and infrastructure.

Since M2X ecosystems are a source of large, unstructured data sets that must be combined and understood to extract intelligence with advanced analytic for actionable decision-making, it is our contention that trust management is only possible with novel blockchain technology of high scalability and performance. For example, the use of blockchains in a M2X ecosystem involves many devices that have low storage capacity and computing power. Since these devices cannot maintain a blockchain of many gigabytes, novel sharding management for blockchain parts to and from devices is required to overcome storage and computing-power limitations [1,57].

\subsection{Tokenized Value Exchange}

A blockchain-based solution enables the decentralized settlement of value added in the form of crypto tokens [26,58]. The latter may be created entirely without trusted third parties, or intermediaries and exchanged directly P2P [53] while at the same time increasing transaction speed. Since Section 4 stipulates that the legacy financial technologies with a focus on fiat currencies is not suitable and lacks the required utility for the M2X Economy, we put forward further arguments that justify the need for a smart-contract blockchain based token economy. Given the legal and socio-technical complexity of a M2X Economy, it is essential to have a flexible monetary instrument that allows for flexibility with respect to defining for a token the application goals, the properties, the business, and incentivizing governance models. Important for the development of a token model with a specific degree of $\mathrm{M} 2 \mathrm{X}$ required complexity is to also target in that process the desired legal-compliance adjustment. Certainly for tokens with a high degree of contextual application complexity, e.g., to tackle governance issues in a M2X Economy, the business-model engineering gains in dominance additionally to legal-compliance assurance.

To expand on the topic of e-governance by tokens, essential for this is the provision of a rich and real-time availability of large data sets stemming from the entities that comprise a M2X Economy. Smart-contract blockchain tokens pose via their incentivized transaction involvement that they facilitate the generation of such data with all economic action involved. With all that, the scope emerges for establishing a novel scientific discipline that may be termed economic systems engineering. Thus, diverse economics and engineering disciplines need to be combined in this novel scientific discipline for M2X Economics in which blockchain-specific consensus mechanisms such as PoW allow for a real-time steering of complex governance scenarios in a trustless collaboration context of complex and adaptive M2X Economies where all services are tokenized themselves. 


\section{Conclusions and Future Work}

This position paper argues for a novel business model for the emerging M2X Economy of multi-stakeholders that is open, decentralized, and distributed. As such, the M2X Economy encompasses the interactions between smart autonomous devices with other machines, humans and infrastructure in a cybernetic context. As an example, we correspondingly present a running case from the domain of self-driving autonomous smart vehicles to be rented by humans for transportation on roads with smart toll gates and smart traffic lights in interaction with other smart vehicles.

Important supporting concepts for the M2X Economy are lifecycle management for the setup, establishment, rollout, rollback and orderly termination of business collaborations. This lifecycle manages cross-organizational process-aware collaboration establishment that is expressed in machine-readable smart contracts.

The suggested course of actions for developing the M2X Economy needs to focus on specific domains. First, since smart contracts are a promising means for managing ad-hoc P2P contractual collaboration establishment, it is important to develop smart-contract languages that have legal relevance with their representation in a machine-readable format. Important is in this context that openness and interoperability must be assured to avoid self-contained data silos and instead enable collaboration transparency for effortless conflictresolution e-governance mechanisms. Next, an M2X Economy requires the adoption of novel identity authentication for the participating entities and humans that are flexible in the adoption of application-context adjusted challenge sets. Thereby considering scalable and highly performing blockchain technology, a trusted entry into and exit from an M2X ecosystem can be assured for smart autonomous devices, machines, infrastructure and humans. Finally, an M2X Economy should have its incentive mechanisms governed by programmable, smart token sets that are developed with means of smart-contract blockchain technologies.

Exploring the solution options, we observe that smart contracts still lack legal relevance due to missing language contracts. For example, traditional contracts are based on the formulation of obligations and rights that should be part of smart contracts in a machine-readable form. To achieve openness and interoperability for an M2X Economy, the lack of standards that technology providers adhere to should be addressed. For addressing the topic of suitable identity-authentication mechanisms, we claim that the investigation of application-context dependent multi-factor challenge sets are a promising means for trusted entries and exits of humans and non-human actors into a M2X ecosystem. A novel generation of blockchains with scaling and performing consensus algorithms is essential to assure effective trust assurance by investigating novel distributed blockchain-sharding management. Finally, the need arises for establishing economic systems engineering as a scientific discipline for investigating the important domain of tokenized M2X value exchanges.

Author Contributions: Conceptualization, B.L., P.S. and A.N.; Writing-original draft, B.L., P.S. and A.N.; Writing-review \& editing, B.L., P.S. and A.N. All authors have read and agreed to the published version of the manuscript.

Funding: We acknowledge financial support by Open Access Publishing Fund of Clausthal University of Technology.

Data Availability Statement: Not Applicable, the study does not report any data.

Conflicts of Interest: The authors declare no conflict of interest. 


\section{References}

1. Leiding, B. The M2X Economy-Concepts for Business Interactions, Transactions and Collaborations Among Autonomous Smart Devices. Ph.D. Thesis, University of Göttingen, Göttingen, Germany, 2020. Available online: http://hdl.handle.net/21.11130/001735-0000-0005-12E4-5 (accessed on 30 October 2021).

2. Männi, M. Tallinn's New Self-Driving Bus Emerged from a University Robotics Course. 2020. Available online: https: / / estonianworld.com/technology/tallinns-new-self-driving-bus-emerged-from-a-university-robotics-course (accessed on 29 September 2021).

3. Bellan, R. Starship Technologies Is Bringing Food Delivery Robots to Four More US College Campuses This Year. 2021. Available online: https: / / techcrunch.com/2021/08/10/starship-technologies-is-bringing-food-delivery-robots-to-four-more-us-collegecampuses-this-year (accessed on 18 October 2021).

4. Lynggaard, P.; Skouby, K. Complex IoT Systems as Enablers for Smart Homes in a Smart City Vision. Sensors 2016, 16, 1840. [CrossRef] [PubMed]

5. Vaidya, S.; Ambad, P.; Bhosle, S. Industry 4.0-A Glimpse. Procedia Manuf. 2018, 20, 233-238. [CrossRef]

6. Savaget, P.; Geissdoerfer, M.; Kharrazi, A.; Evans, S. The Theoretical Foundations of Sociotechnical Systems Change for Sustainability: A Systematic Literature Review. J. Clean. Prod. 2019, 206, 878-892. [CrossRef]

7. Leiding, B.; Vorobev, W.V. Enabling the V2X Economy Revolution Using a Blockchain-based Value Transaction Layer for Vehicular Ad-hoc Networks. In Proceedings of the 12th Mediterranean Conference on Information Systems-MCIS, Corfu, Greece, 28-30 September 2018.

8. Udokwu, C.; Kormiltsyn, A.; Thangalimodzi, K.; Norta, A. The State of the Art for Blockchain-Enabled Smart-Contract Applications in the Organization. In Proceedings of the 2018 Ivannikov Ispras Open Conference (ISPRAS), Moscow, Russia, 22-23 November 2018; pp. 137-144.

9. Wiener, N. Cybernetics or Control and Communication in the Animal and the Machine; MIT Press: Cambridge, MA, USA, 2019.

10. Wan, J.; Chen, M.; Xia, F.; Di, L.; Zhou, K. From Machine-to-Machine Communications Towards Cyber-Physical Systems. Comput. Sci. Inf. Syst. 2013, 10, 1105-1128. [CrossRef]

11. Cyber-Physical Systems (CPS) I NSF-National Science Foundation, nsf19553. 2019. Available online: https://www.nsf.gov/ publications /pub_summ.jsp?ods_key=nsf19553 (accessed on 7 September 2021).

12. Gubbi, J.; Buyya, R.; Marusic, S.; Palaniswami, M. Internet of Things (IoT): A Vision, Architectural Elements, and Future Directions. Future Gener. Comput. Syst. 2013, 29, 1645-1660. [CrossRef]

13. Madakam, S.; Holmukhe, R.M.; Jaiswal, D.K. The Future Digital Work Force: Robotic Process Automation (RPA). JISTEM-J. Inf. Syst. Technol. Manag. 2019, 16. [CrossRef]

14. Hofmann, P.; Samp, C.; Urbach, N. Robotic Process Automation. Electron. Mark. 2020, 30, 99-106. [CrossRef]

15. Khaitan, S.K.; McCalley, J.D. Design Techniques and Applications of Cyberphysical Systems: A Survey. IEEE Syst. J. 2015, 9, 350-365. [CrossRef]

16. Gunes, V.; Peter, S.; Givargis, T.; Vahid, F. A Survey on Concepts, Applications, and Challenges in Cyber-Physical Systems. KSII Trans. Internet Inf. Syst. 2014, 8, 4242-4268.

17. Shi, J.; Wan, J.; Yan, H.; Suo, H. A Survey of Cyber-Physical Systems. In Proceedings of the 2011 International Conference on Wireless Communications and Signal Processing (WCSP), Nanjing, China, 9-11 November 2011; pp. 1-6.

18. Da Xu, L.; He, W.; Li, S. Internet of Things in Industries: A Survey. IEEE Trans. Ind. Informatics 2014, 10, $2233-2243$.

19. Al-Fuqaha, A.; Guizani, M.; Mohammadi, M.; Aledhari, M.; Ayyash, M. Internet of Things: A Survey on Enabling Technologies, Protocols, and Applications. IEEE Commun. Surv. Tutorials 2015, 17, 2347-2376. [CrossRef]

20. Monostori, L.; Kádár, B.; Bauernhansl, T.; Kondoh, S.; Kumara, S.; Reinhart, G.; Sauer, O.; Schuh, G.; Sihn, W.; Ueda, K. Cyber-Physical Systems in Manufacturing. Cirp Ann. 2016, 65, 621-641. [CrossRef]

21. Ivančić, L.; Vugec, D.S.; Vukšić, V.B. Robotic Process Automation: Systematic Literature Review. In Proceedings of the International Conference on Business Process Management, Vienna, Austria, 1-6 September 2019; Springer: Berlin/Heidelberg, Germany, 2019; pp. 280-295.

22. Roxenhall, T.; Ghauri, P. Use of the Written Contract in Long-lasting Business Relationships. Ind. Mark. Manag. 2004, 33, 261-268. [CrossRef]

23. Norta, A. Designing a Smart-Contract Application Layer for Transacting Decentralized Autonomous Organizations. In Proceedings of the International Conference on Advances in Computing and Data Sciences, Ghaziabad, India, 11-12 November 2016; Springer: Berlin/Heidelberg, Germany, 2016; pp. 595-604.

24. Szabo, N. Smart Contracts. 1994. Available online: http://www.fon.hum.uva.nl/rob/Courses/InformationInSpeech/CDROM/ Literature/LOTwinterschool2006/szabo.best.vwh.net/smart.contracts.html (accessed on 2 October 2021).

25. Szabo, N. The Idea of Smart Contracts. 1997. Available online: http://www.fon.hum.uva.nl/rob/Courses/InformationInSpeech/ CDROM/Literature/LOTwinterschool2006/szabo.best.vwh.net/smart_contracts_idea.html (accessed on 2 October 2021).

26. Nakamoto, S. Bitcoin: A Peer-to-Peer Electronic Cash System. 2008. Available online: https://bitcoin.org/bitcoin.pdf (accessed on 9 September 2021).

27. Kutvonen, L.; Norta, A.; Ruohomaa, S. Inter-Enterprise Business Transaction Management in Open Service Ecosystems. In Proceedings of the 2012 IEEE 16th International Enterprise Distributed Object Computing Conference, Beijing, China, 10-14 September 2012; pp. 31-40. 
28. Eshuis, R.; Norta, A.; Roulaux, R. Evolving process views. Inf. Softw. Technol. 2016, 80, 20-35. [CrossRef]

29. Badawy, M.M.; Ali, Z.H.; Ali, H.A. QoS provisioning framework for service-oriented internet of things (IoT). Clust. Comput. 2020, 23, 575-591. [CrossRef]

30. Eshuis, R.; Norta, A.; Kopp, O.; Pitkänen, E. Service outsourcing with process views. IEEE Trans. Serv. Comput. 2013, 8, 136-154. [CrossRef]

31. Norta, A.; Grefen, P.; Narendra, N.C. A reference architecture for managing dynamic inter-organizational business processes. Data Knowl. Eng. 2014, 91, 52-89. [CrossRef]

32. Ruokolainen, T.; Ruohomaa, S.; Kutvonen, L. Solving Service Ecosystem Governance. In Proceedings of the 2011 IEEE 15th International Enterprise Distributed Object Computing Conference Workshops, Helsinki, Finland, 29 August-2 September 2011; pp. $18-25$.

33. Dwivedi, V.; Pattanaik, V.; Deval, V.; Dixit, A.; Norta, A.; Draheim, D. Legally Enforceable Smart-Contract Languages: A Systematic Literature Review. ACM Comput. Surv. (CSUR) 2021, 54, 1-34. [CrossRef]

34. Dwivedi, V.; Norta, A.; Wulf, A.; Leiding, B.; Saxena, S.; Udokwu, C. A Formal Specification Smart-Contract Language For Legally Binding Decentralized Autonomous Organizations. IEEE Access 2021, 9, 76069-76082. [CrossRef]

35. Dwivedi, V.; Norta, A. A Legal-Relationship Establishment in Smart Contracts: Ontological Semantics for Programming-Language Development. In Proceedings of the International Conference on Advances in Computing and Data Sciences, Nashik, India, 23-24 April 2021; Springer: Berlin/Heidelberg, Germany, 2021; pp. 660-676.

36. Norta, A.; Kutvonen, L. A cloud hub for brokering business processes as a service: A "rendezvous" platform that supports semi-automated background checked partner discovery for cross-enterprise collaboration. In Proceedings of the 2012 Annual SRII Global Conference, San Jose, CA, USA, 24-27 July 2012; pp. 293-302.

37. Petri, C.A.; Reisig, W. Petri net. Scholarpedia 2008, 3, 6477. [CrossRef]

38. Norta, A.; Ma, L.; Duan, Y.; Rull, A.; Kõlvart, M.; Taveter, K. eContractual Choreography-language Properties Towards Cross-organizational Business Collaboration. J. Internet Serv. Appl. 2015, 6, 1-23. [CrossRef]

39. Lee, J.Y. A Decentralized Token Economy: How Blockchain and Cryptocurrency can Revolutionize Business. Bus. Horizons 2019, 62, 773-784. [CrossRef]

40. Di Angelo, M.; Salzer, G. Tokens, Types, and Standards: Identification and Utilization in Ethereum. In Proceedings of the 2020 IEEE International Conference on Decentralized Applications and Infrastructures (DAPPS), Oxford, UK, 3-6 August 2020; pp. $1-10$.

41. Udokwu, C.; Anyanka, H.; Norta, A. Evaluation of Approaches for Designing and Developing Decentralized Applications on Blockchain. In Proceedings of the 2020 4th International Conference on Algorithms, Computing and Systems, Rabat, Morocco, 6-8 January 2020; pp. 55-62.

42. Udokwu, C.; Norta, A. Deriving and Formalizing Requirements of Decentralized Applications for Inter-Organizational Collaborations on Blockchain. Arab. J. Sci. Eng. 2021, 46, 8397-8414. [CrossRef]

43. Norta, A.; Rossar, R.; Parve, M.; Laas-Billson, L. Achieving a High Level of Open Market-Information Symmetry with Decentralised Insurance Marketplaces on Blockchains. In Intelligent Computing-Proceedings of the Computing Conference, London, UK, 16-17 July 2019; Springer: Berlin/Heidelberg, Germany, 2019; pp. 299-318.

44. Norta, A.; Hawthorne, D.; Engel, S.L. A Privacy-Protecting Data-Exchange Wallet with Ownership-and Monetization Capabilities. In Proceedings of the 2018 International Joint Conference on Neural Networks (IJCNN), Rio de Janeiro, Brazil, 8-13 July 2018; pp. 1-8.

45. Bamakan, S.M.H.; Motavali, A.; Bondarti, A.B. A Survey of Blockchain Consensus Algorithms Performance Evaluation Criteria. Expert Syst. Appl. 2020, 154, 113385. [CrossRef]

46. Geiger, M.; Harrer, S.; Lenhard, J.; Wirtz, G. BPMN 2.0: The State of Support and Implementation. Future Gener. Comput. Syst. 2018, 80, 250-262. [CrossRef]

47. Levy, M. Agent-Based Computational Economics. In Complex Social and Behavioral Systems: Game Theory and Agent-Based Models; 2020; pp. 825-849. Available online: https://link.springer.com/referenceworkentry/10.1007\%2F978-1-0716-0368-0_6 (accessed on 12 December 2021).

48. Olsen, M. How Firms Overcome Weak International Contract Enforcement: Repeated Interaction, Collective Punishment and Trade Finance. In IESE Business School Working Paper No. WP-1111-E; 2016. Available online: https://www.morten-olsen.com/ Morten\%20Olsen\%20-\%20how\%20firms\%20overcome\%20weak\%20contract.pdf (accessed on 12 December 2021).

49. Timmer, I. Contract Automation: Experiences from Dutch Legal Practice. In Legal Tech, Smart Contracts and Blockchain; Springer: Berlin/Heidelberg, Germany, 2019; pp. 147-171.

50. Wood, G. Ethereum: A Secure Decentralized Generalised Transaction Ledger. 2014. Available online: http://gavwood.com/ paper.pdf (accessed on 13 October 2021).

51. Buterin, V. A Next-Generation Smart Contract and Decentralized Application Platform-Whitepaper. 2019. Available online: https://github.com/ethereum/wiki/wiki/White-Paper (accessed on 14 October 2021).

52. Wood, G. Polkadot: Vision for a Heterogenous Multi-Chain Framework-Draft 1 (White Paper). 2016. Available online: https:/ / polkadot.network/PolkaDotPaper.pdf (accessed on 8 September 2021).

53. Cap, C.H.; Leiding, B. Blogchain-Disruptives Publizieren auf der Blockchain. HMD Prax. Wirtsch. 2018, 55, 1326-1340. [CrossRef] 
54. Othman, A.; Callahan, J. The Horcrux Protocol: A Method for Decentralized Biometric-based Self-Sovereign Identity. In Proceedings of the 2018 International Joint Conference on Neural Networks (IJCNN), Rio de Janeiro, Brazil, 8-13 July 2018; pp. 1-7.

55. Sporny, M.; Longley, D.; Sabadello, M.; Drummond, R.; Steelie, O.; Allen, C. Decentralized Identifiers (DIDs)-Core Architecture, Data Model, and Representations v1.0. 2021. Available online: https://w3c-ccg.github.io/did-spec/ (accessed on 14 October 2021).

56. Zhou, Q.; Huang, H.; Zheng, Z.; Bian, J. Solutions to Scalability of Blockchain: A Survey. IEEE Access 2020, 8, 16440-16455. [CrossRef]

57. Dang, H.; Dinh, T.T.A.; Loghin, D.; Chang, E.C.; Lin, Q.; Ooi, B.C. Towards Scaling Blockchain Systems via Sharding. In Proceedings of the 2019 International Conference on Management of Data, Amsterdam, The Netherlands, 30 June-5 July 2019 ; pp. 123-140.

58. Schär, F. Decentralized Finance: On Blockchain and Smart Contract-based Financial Markets. In FRB of St. Louis Review; 2021. Available online: https:/ / papers.ssrn.com/sol3/papers.cfm?abstract_id=3843844 (accessed on 12 December 2021). 